\title{
Optical properties of diffuse ZnSe:Mn layers
}

\author{
Makhniy V.P., Kinzerskaya O.V., Horley P.P. and Ul’yanitskiy K.S. \\ Yuri Fedkovych Chernivtsi National University, 2 Kotsyubynsky St., \\ 58012 Chernivtsi, Ukraine, e-mail: OksanaKinzersky@ rambler.ru
}

Received: 22.11.2007

\begin{abstract}
It is shown that doping of zinc selenide with $\mathrm{Mn}$ atoms from the vapour phase causes appearance of the band peaked at $\hbar \omega \approx 2.3 \mathrm{eV}$ in the luminescence and transmission spectra. The intensity of this band changes with the temperature of diffusion annealing and varies after introduction of other doping impurities to the basic substrate, which could be explained by increased solubility of Mn atoms.
\end{abstract}

Keywords: zinc selenide, magnetic impurity, photoluminescence, transmission

PACS: 78.55.Et

UDC: 535.37

\section{Introduction}

Semiconductor materials can be successfully used in functional electronics if they have specific physical and technical parameters required within a given application field. The proper values of some of these parameters may be achieved while creating a specific ensemble of point defects, which is usually formed owing to introduction of certain doping elements during the crystal growth. However, this approach may not always function for the bulk crystals of II-VI compounds. In some specific cases it is difficult, or even impossible, to obtain the crystals with required type and magnitude of conductivity, set the generation-recombination processes into a desired spectral range, obtain some exactly determined concentration of impurities, etc. [1]. To avoid these disadvantages, it is imperative to perform additional technological after-crystal-growth operations, in particular to use the diffusion methods [2]. The latter allow changing the properties of the bulk crystals in quite a simple way, suitable also for the formation of thin layers used in the devices based on the wide-band semiconductor materials with a direct-band structure.

It is also worth mentioning that the thin layers are extremely important for many spintronic applications, among which one may often find the rectifying structures such as metal / diluted magnetic semiconductor, magnetic metal / semiconductor, etc. [3]. In order to produce the corresponding structures based on II-VI compounds, which have good and stable parameters, one needs to solve a particular set of problems, with the most important one concerning a proper choice of methods employed for doping the material with magnetic impurities. The second problem consists in lowering concentration of defects at the substrate surface, since the latter act usually as efficient trapping and recombination 
centres, thus worsening parameters of the rectifying structure.

This work is dedicated to one of promising technologies, which enables one to avoid the aforementioned difficulties in obtaining of thin ZnSe:Mn layers. We also report the experimental results on a number of optical properties of the latter.

\section{Experimental details}

The basic substrates were made in the form of plates with the dimensions $4 \times 4 \times 1 \mathrm{~mm}^{3}$, cut from single crystals of zinc selenide of the two types. The first ones were obtained with the Markov-Davydov method from the vapour phase of stoichiometric content and the samples of the second type were grown with the Bridgman method from the relevant melt under pressure of a noble gas and doped with $\mathrm{Al}$ during crystal growth up to the concentration of 0.1 molar per cent. Further on, we distinguish these crystals by calling them as $\mathrm{ZnSe}$ and $\mathrm{ZnSe}<\mathrm{Al}>$, respectively.

The substrates were subjected to both mechanical and chemical polishing, with careful rinsing in de-ionized water. After the processing the substrates featured mirror-smooth surface and displayed bulk luminescence. The doping was performed in pumped out (to $10^{-4}$ Torr) and sealed quartz container, with the substrate and the doping material positioned at its opposite ends. The process was carried out for several hours in isothermal conditions at the temperatures $T_{a}=900-1200^{\circ} \mathrm{C}$. To prevent surface erosion of the plate, the containers were loaded with elementary Se, in addition to Mn.

Our preliminary experiments showed that the manganese interacts strongly with quartz at $T_{a} \geq 1000^{\circ} \mathrm{C}$, which can cause damage to the containers during the diffusion process. To avoid this effect, we covered the inner surface of the container with a thin pyro-graphite layer around the doping element. Several annealed plates of $\mathrm{ZnSe}$ were additionally treated with a special etching solution so that their surface became dim due to formation of quantum-scale texture [4]. We would address the samples with the mirrorlike surface as samples of type 1 and those with the dim surface as samples of type 2, respectively.

The optical transmission and photoluminescence (PL) spectra of the samples were measured at $300 \mathrm{~K}$, involving a commonly used technique and a standard synchronousdetection scheme. The spectral measurements were performed with a diffraction monochromator MDR-23, using a xenon lamp and a nitrogen laser (the wavelength of $\lambda_{m} \approx 0.337 \mu \mathrm{m}$ ) as radiation sources. All the optical spectra were recorded automatically, taking into account nonlinearity of components of the measurement system. The PL spectra were plotted in the coordinates $N_{\omega}$ (the number of quanta per the unit energy range) versus $\hbar \omega$ (the energy of quantum).

\section{Results and discussion}

Our results have proven significant decrease of the transmission coefficient $T_{\omega}$ for both the initial and annealed $\mathrm{ZnSe}$ substrates with the dim surface, comparing with those with the mirror-like one (see Fig. 1). This can be explained by light scattering at the surface 
precipitates created by sample etching. At the same time, the absence of a shift of highenergy edge of $T_{\omega}$ spectrum towards low energies indicates that the lateral dimensions of the surface inhomogeneities do not exceed $50 \mathrm{~nm}[4,5]$.

$$
\mathrm{T}_{\omega} \text {, a. u. }
$$

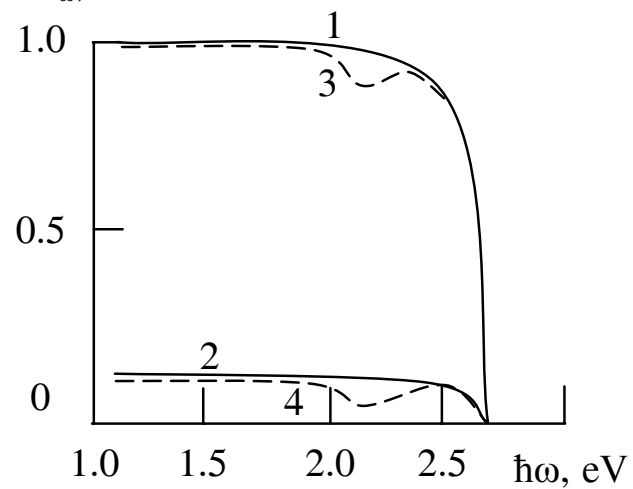

Fig. 1. Optical transmission spectra for the initial (curves 1,2) and annealed (curves 3 , 4) substrates of $\mathrm{ZnSe}$ with the mirrorreflective (curves 1,3) and dim (curves 2, 4) surfaces at $300 \mathrm{~K}$.

It is worth a special attention that the transmission spectra of the samples annealed in Mn vapours include a singularity at $\hbar \omega \approx 2.3 \mathrm{eV}$, which is absent in the initial substrates of both types (see Fig. 1). The amplitude of this singularity increases for higher annealing temperatures. Therefore it has to be just the Mn impurity that is responsible for its formation.

Our studies have shown that the shape of the emission spectra and the intensity of the corresponding peaks depend significantly on such technological factors as the composition of intrinsic and impurity defects, the diffusion temperature, the technique used for the surface treatment, etc. Nonetheless, detailed additional studies are required for formulating general tendencies of those dependences.

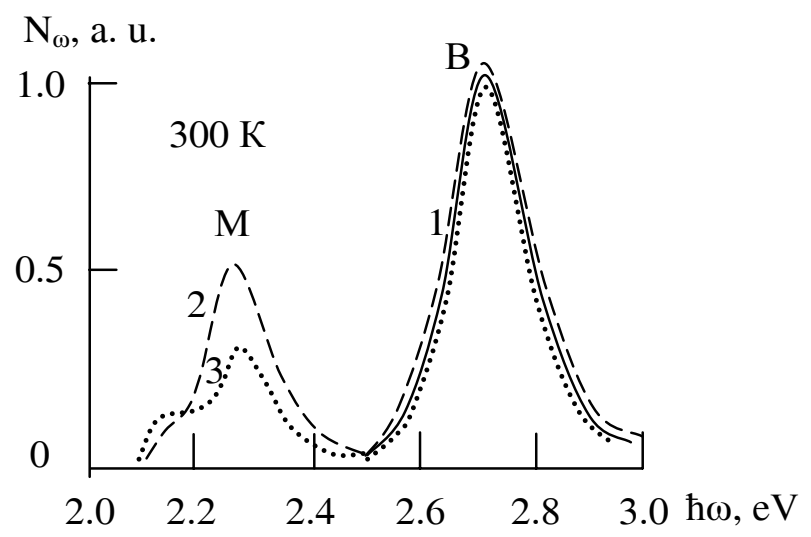

Fig. 2. Luminescence spectra for the substrate ZnSe with the mirror surface (1) and ZnSe:Mn layers (curves 2, 3) for different excitation levels: curves $1,2-10^{18}$ and curve $3-10^{16}$ quanta per second.

It has been found experimentally that doping with Mn leads to formation of an extra band in the luminescence spectra, which we refer to as "M-band". It has a peak located at $\hbar \omega_{m} \approx 2.3 \mathrm{eV}$, irrespective of type of the basic substrate. This effect is more pronounced in the diffuse layers of ZnSe:Mn (see Fig. 2). We should notice that the emission spectra of $\mathrm{ZnSe}<\mathrm{Al}>\mathrm{Mn}$ layers manifest more complicated behaviour in this energy range, though the intensity of the M-band for these samples is significantly higher than that of 
ZnSe:Mn. The peculiarities mentioned above are, most probably, caused by a higher content of defects in the doped crystals, comparing to that typical for the undoped crystals. Larger number and different nature of point defects result in the complicated emission spectra. On the other hand, these factors favour localization of $\mathrm{Mn}$ atoms in the vicinity of the same defects, increasing solubility of the doping impurity. The similar effect has also been reported in the work [6] basing on the EPR studies of $\mathrm{ZnSe}$ and $\mathrm{ZnS}$ crystals doped with magnetic impurities during the growth process.

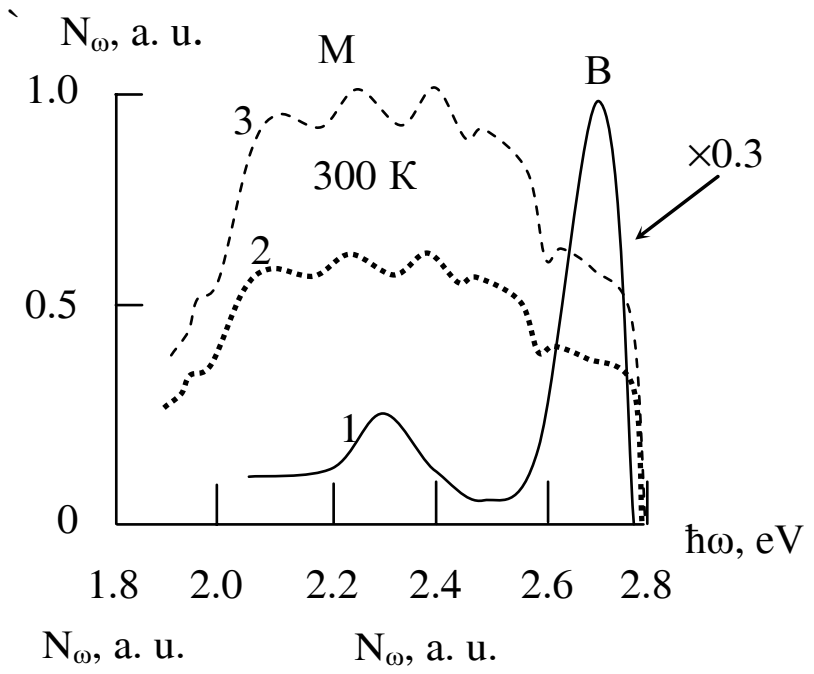

Fig. 3. Luminescence spectra for $\mathrm{ZnSe}<\mathrm{Al}>\mathrm{Mn}$ sample obtained at 1000 (curve 1), 1100 (2) and $1200^{\circ} \mathrm{C}(3)$.

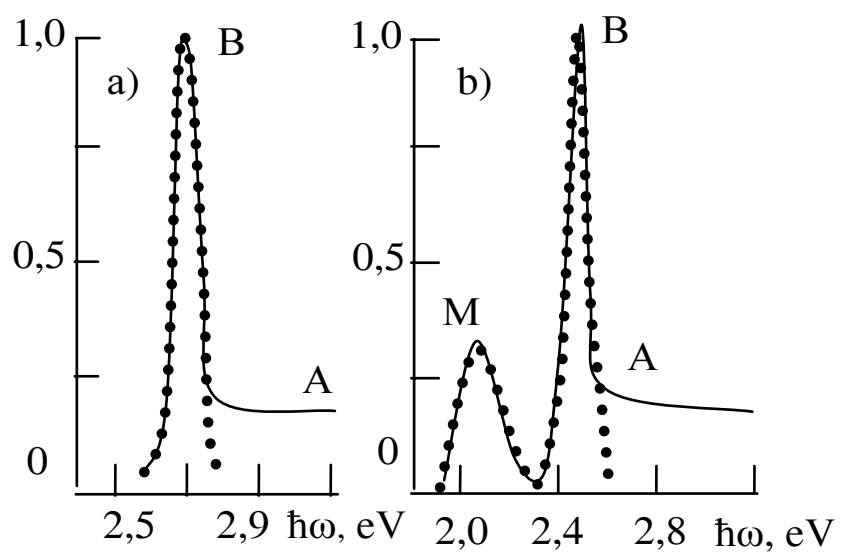

Fig. 4. Luminescence spectra for ZnSe substrates (a) and ZnSe:Mn layers (b) with the mirror (circles) and dim (solid lines) surfaces at $300 \mathrm{~K}$.

Decrease in the excitation level stipulates formation of another peak at $\hbar \omega_{m} \approx 2.1 \mathrm{eV}$, which is illustrated in Fig. 2 for the layers deposited on $\mathrm{ZnSe}$ substrates. The correctness of attribution of the M-band to just the manganese but not the other impurity or the point defect is evidenced by the absence of this peak in the initial crystals of both types. On the other hand, according to the study [7], annealing of undoped ZnSe substrates in vacuum or vapours of Se (the element appearing in the furnace charge) gives rise to increase in the edge emission, though does not form any band positioned at $\hbar \omega_{m} \approx 2.3 \mathrm{eV}$. Finally, there is no singularity at the energies about $2.3 \mathrm{eV}$ in the transmission spectra of the samples. 
It is natural to assume that increase in the annealing temperatures would evoke higher intensity of the M-band due to larger solubility of manganese ions. It is also known that the manganese contained in II-VI compounds substitutes the metal atoms, so that it usually appears in the charged state $\mathrm{Mn}^{2+}$ [6]. This is evidenced experimentally for the case of $\mathrm{ZnSe}<\mathrm{Al}>$ crystals (see Fig. 3). As follows from the latter, contribution of the M-band to the emission spectra of annealed samples becomes significant for $T_{a} \geq 1000^{\circ} \mathrm{C}$, determining the lowest possible annealing temperature. The similar data have also been obtained for the undoped $\mathrm{ZnSe}$ substrates.

It is important that the $\mathrm{M}$-band also appears in $\mathrm{ZnO}$ heterolayers formed by isovalent substitution on the ZnSe:Mn substrates [8]. On the one hand, this fact emphasizes once more the peculiarities of mechanism of the isovalent substitution [9] and, on the other hand, proves that the inner-centre radiative transitions taking place inside $\mathrm{Mn}$ ions participate in formation of the M-band. At the same time, the peaks at 2.3 and $2.1 \mathrm{eV}$ correspond to the highest and the neighbouring lower excitation levels of Mn ion.

The PL of all the samples with the dim surface (i.e., the samples of type 2 ) is characterized by the emission with the quantum energies exceeding the bandgap of zinc selenide $\left(E_{g}=2.7 \mathrm{eV}\right.$; see the A-band in Fig. 4).

According to the works $[4,5]$, a notable width and lack of any structure for the Aband are caused by significant dispersion of both the dimensions and shapes of surface inhomogeneities. The presence of photons with $\hbar \omega>E_{g}$ should be related to quantumscale effects. It is also worthwhile to mention that the intensity of the edge emission (denoted as the B-band in figures) for the samples of type 2 is several times larger than that for the samples of type 1 . This does not contradict the data presented in the studies [5, 10] concerning a considerable decrease in the surface recombination rate owing to formation of quantum-scale structure.

\section{Conclusions}

The results presented in this work prove that there is a possibility to perform doping of zinc selenide with the magnetic impurity of Mn, using diffusion from the vapour phase. Formation of the M-band in the transmission and PL spectra at the energies $\sim 2.3 \mathrm{eV}$ may be attributed to the manganese ions, because the intensity of this band increases for higher diffusion temperatures. Higher intensities of the M-band for $\mathrm{ZnSe}<\mathrm{Al}>$ :Mn samples, in comparison with those of ZnSe:Mn, may be explained by larger solubility of the manganese atoms in the material possessing higher concentrations of the point defects.

This study has been partially supported by the STCU Project No. 3098.

\section{References}

1. Georgiobiany A. N. and Sheinkman M. K. Physics of $\mathrm{A}^{\mathrm{II}} \mathrm{B}^{\mathrm{VI}}$ compounds. Moscow: Nauka (1986).

2. Pichugin I. G. and Tairov Yu. M. Technology of semiconductor devices. Moscow: Vysshaya shkola (1984).

Ukr. J. Phys. Opt. 2008, V9, №2 
3. Dietl T. Spintronics And Ferromagnetism In Wide-Band-Gap Semiconductors, 27 Int. Conf. on the Phys. of Semicon./ Americ. Inst. of Phys. (2005) p. 56-61.

4. Makhniy V P, Demich M V, and Slyotov M M, Gorley P P, Vorobiev Yu V and Gonzalez-Hermandez J, 2006. Optical properties of cadmium selenide heterostructures with quantum-scale surface formation. Thin Solid Films. 495: 372-374.

5. Makhniy V P, 2005. Specific features of the physical properties of a modified CdTe surface. Semiconductors. 39: 792-794.

6. Bulanyj M.F., Kovalenko O.V., Omel'chenko S.O. and Shtambur I.V. Resonance phenomena. Dnipropetrovs'k: Art-Press. (2006).

7. Tkachenko I.V. Mechanisms of defect formations and luminescence in undoped and tellurium doped crystals of zinc selenide. Dissertation for Ph.D. degree in physics and mathematics. Chernivtsi (2005).

8. Makniy V.P., Melnyk V.V., Sletov M.M., Kinzerskaya O.V., Cvikl B., Korosak D., Gorley P.N. and Horley P.P. Optical properties of ZnO:Mn heterolayers with quantum-scale surface formations. Proc. 43rd Intern. Conf. on Microelectronics, Devices and Materials. (2007) p. 85-87.

9. Baranyuk V Yel, Demich M V, Makhnyi V P, Melnik V V, Malimon I V, Slyotov M M, Sobistchanskiy B M and Stets E V, 2000. Isovalent substitution: a perspective method of producing heterojunction optoelectronical devices. Proc. SPIE 4425: 272 277.

10. Makhniy V.P., Demych M.V. and Slyotov M.M., Patent of Ukraine No.65010A, published 15.03.2004. 